\title{
Robust Control, Optimization, and Applications to Markovian Jumping Systems
}

\author{
Shuping He, ${ }^{1}$ Zhengguang $\mathrm{Wu},{ }^{2}$ Hao Shen, ${ }^{3}$ Yanyan Yin, ${ }^{4}$ and Quanxin $\mathrm{Zhu}^{5}$ \\ ${ }^{1}$ School of Electrical Engineering and Automation, Anhui University, Hefei 230601, China \\ ${ }^{2}$ National Laboratory of Industrial Control Technology, Institute of Cyber-Systems and Control, Zhejiang University, \\ Hangzhou 310058, China \\ ${ }^{3}$ School of Electrical and Information Engineering, Anhui University of Technology, Ma'anshan 243002, China \\ ${ }^{4}$ Department of Mathematics and Statistics, Curtin University, Perth, WA 00301J, Australia \\ ${ }^{5}$ School of Mathematical Sciences, Nanjing Normal University, Nanjing 210023, China \\ Correspondence should be addressed to Shuping He; shuping.he@ahu.edu.cn
}

Received 27 May 2014; Accepted 27 May 2014; Published 24 June 2014

Copyright (C) 2014 Shuping He et al. This is an open access article distributed under the Creative Commons Attribution License, which permits unrestricted use, distribution, and reproduction in any medium, provided the original work is properly cited.

Markovian jumping systems have arisen naturally in the mathematical modeling of phenomena spanning disciplines in the social sciences, natural sciences, and engineering. This kind of stochastic dynamical systems can be employed to model the dynamics when parameters are subject to random abrupt changes due to sudden environment changes, subsystem switching, system noises, executor faults, and so forth. Much attention has been given to modeling, optimization, and real applications of such stochastic dynamical systems in the literature in recent years. As the advanced control and optimization will provide a basis for the design and application of such stochastic systems, these advanced techniques would result in substantial and sustainable benefits. The accepted papers in this special issue include stochastic stability, stabilization, stochastic control optimization, system modeling and identification methods, predictive control, signal processing, robust filtering, multiagent systems, networked control systems, time-delayed systems, neural networks, the Takagi-Sugeno fuzzy systems, simulated annealing, and fault detection methods.

We have accepted thirty-six papers in this special issue. In the published papers, eight consider the stability and stabilization problems of stochastic systems. There are fourteen papers which discuss the problems of the controller design and relevant optimization algorithms. Six articles study the system modeling and identification methods. One paper focuses on the fault detection for wireless networked control systems with stochastic uncertainties and multiple time delays, and seven consider the state estimation and filtering problems.

The problems of stochastic stability and stabilization problems of Markovian jumping systems have been extensively studied by many researchers, and many relevant results have been made. The paper entitled "Sufficient conditions on the exponential stability of neutral stochastic differential equations with time-varying delays" by Y. Tian and B. Chen considers the exponential stability in almost sure sense of the neutral stochastic differential equations with timevarying delays and the paper entitled "Delay-dependent robust exponential stability and $H_{\infty}$ analysis for a class of uncertain Markovian jumping system with multiple delays" by J. Xia deals with the problem of robust exponential stability and $H_{\infty}$ performance analysis for a class of uncertain Markovian jumping systems with multiple delays. The paper entitled "On input-to-state stability of impulsive stochastic systems with time delays" by F. Yao et al. is concerned with pth moment input-to-state stability and stochastic input-to-state stability of impulsive stochastic systems with time delays. The paper entitled "Absolute stability of a class of nonlinear singular systems with time delay" by H.-B. Zeng et al. studies the absolute stability for a class of nonlinear singular systems with time delay. The paper entitled "Analysis and design of 
networked control systems with random Markovian delays and uncertain transition probabilities" by L. Qiu et al. focuses on the stability issue of discrete-time networked control systems with random Markovian delays and uncertain transition probabilities. For the stochastic stabilization aspects, the paper entitled "Finite-time boundedness for a class of delayed Markovian jumping neural networks with partly unknown transition probabilities" by L. Liang is concerned with the problem of finite-time boundedness for a class of delayed Markovian jumping neural networks with partly unknown transition probabilities, and the paper entitled "Output feedback adaptive stabilization of uncertain nonholonomic systems" by Y. Wu et al. investigates the problem of output feedback adaptive stabilization control design for a class of nonholonomic chained systems. The paper entitled "Robust exponential stabilization of stochastic delay interval recurrent neural networks with distributed parameters and Markovian jumping by using periodically intermittent control" by J. Hu et al. considers a class of stochastic delay recurrent neural networks with distributed parameters and Markovian jumping.

In recent years, the research on control optimization for stochastic dynamic systems has received more and more attention. For the $H_{\infty}$ control aspects, the paper entitled "Finite-time $H_{\infty}$ control for discrete-time Markov jump systems with actuator saturation" by B. Li and J. Zhao presents the finite-time control problem for discrete-time Markov jump systems subject to saturating actuators, and the paper entitled "Finite-time $H_{\infty}$ control for a class of discrete-time Markov jump systems with actuator saturation via dynamic antiwindup design" by J. Zhao et al. deals with the finite-time control problem for discrete-time Markov jump systems subject to saturating actuators. The paper entitled "Stochastic finite-time $H_{\infty}$ performance analysis of continuous-time systems with random abrupt changes" by B. Wang proposes the problem of $H_{\infty}$ control performance analysis of continuous-time systems with random abrupt changes. The paper entitled "Robust finite-time $H_{\infty}$ control for nonlinear Markovian jump systems with time delay under partially known transition probabilities" by D. Yang and G. Zong considers the problem of robust finite-time $H_{\infty}$ control for a class of nonlinear Markovian jump systems with time delay under partially known transition probabilities. The paper entitled "Nonfragile $H_{\infty}$ control for stochastic systems with Markovian jumping parameters and random packet losses" by J. Wang and K. Zhang is concerned with the nonfragile $H_{\infty}$ control problem for stochastic systems with Markovian jumping parameters and random packet losses. The paper entitled "Finite-time control for Markovian jump systems with polytopic uncertain transition description and actuator saturation" by $\mathrm{Z}$. Tang addresses the finitetime $L_{2}-L_{\infty}$ control problems for Markovian jump systems with time-varying delays, actuator saturation, and polytopic uncertain transition description; the paper entitled "Resilient robust finite-time $L_{2}-L_{\infty}$ controller design for uncertain neutral system with mixed time-varying delays" by X. Chen and S. He proposes the delay-dependent resilient robust finite-time $L_{2}-L_{\infty}$ control problem of uncertain neutral time-delayed system. For the predictive control aspects, the paper entitled "A simplified predictive control of constrained Markov jump system with mixed uncertainties" by Y. Yin et al. designs a simplified model predictive control algorithm for discretetime Markov jump systems with mixed uncertainties; the paper entitled "Predictive function optimization control for a class of hydraulic servo vibration systems" by X. Feng et al. is concerned with the problem of predictive function control for a class of hydraulic vibration servo control systems. The paper entitled "Global multivariable control of permanent magnet synchronous motor for mechanical elastic energy storage system under multiclass nonharmonic external disturbances" by Y. $\mathrm{Yu}$ et al. proposes a global multivariable control algorithm based on nonlinear internal model principle under multiclass external disturbances; the paper entitled "Feedforward and feedback control performance assessment for nonlinear systems" by Z. Wang and J. Chen proposes a performance assessment method for nonlinear feedforward and feedback control systems. For the multiagent systems control aspects, the paper entitled "Output feedback control for couple-group consensus of multiagent systems" by $\mathrm{H}$. Zhao et al. deals with the couplegroup consensus problem for multiagent systems via output feedback control; the paper entitled "Asynchronous gossipbased gradient-free method for multiagent optimization" by D. Yuan considers the constrained multiagent optimization problem; and the paper entitled "Control of multiagent systems: a stochastic pinning viewpoint" G. Wang develops a stochastic pinning approach for multiagent systems to guarantee such systems being almost surely stable.

Over the past few decades, the signal processing, estimation, and filtering problems have long been the mainstream of research topics. For the filtering problems, the paper entitled "Structural stiffness identification based on the extended Kalman filter research" by F. Wang et al. develops an extended Kalman filter to identify the structural stiffness parameters, the paper entitled "Improved robust $H_{\infty}$ filtering approach for nonlinear systems" by J. Chen and H. Sun presents an improved design approach of robust $H_{\infty}$ filter for a class of nonlinear systems described by the Takagi-Sugeno fuzzy model, and the paper entitled "Delay-dependent robust $L_{2}$ $L_{\infty}$ filtering for a class of fuzzy stochastic systems" by $\mathrm{Z} . \mathrm{Li}$ and X. Yang is concerned with the $L_{2}-L_{\infty}$ filtering problem for a kind of Takagi-Sugeno's fuzzy stochastic system with time-varying delay and parameter uncertainties. For the state estimation problems, the paper entitled "State estimation for wireless network control system with stochastic uncertainty and time delay based on sliding mode observer" by P. Guo et al. considers the state estimation problems for a kind of wireless network control system with stochastic uncertainty and time delay, whereas the paper entitled "State estimation for timedelay systems with Markov jump parameters and missing measurements" by Y. Tan et al. is concerned with the state estimation problem for a class of time-delay systems with Markovian jump parameters and missing measurements. The paper entitled "Constants within error estimates for LegendreGalerkin spectral approximations of control-constrained optimal control problems" by J. Zhou addresses the explicit formulae of constants within a posteriori error estimate for optimal control problems, and the paper entitled "Optimal state estimation for discrete-time Markov jump systems with missing observations" by Q. Sun et al. is concerned with the 
optimal linear estimation for a class of direct-time Markov jump systems with missing observations. The paper entitled "Fault detection for wireless networked control systems with stochastic uncertainties and multiple time delays" by L. Rong et al. investigates the fault detection problem for a class of wireless networked control systems.

As is well known that the system modeling and identification are very useful in engineering applications, the paper entitled "Integration by parts and martingale representation for a Markov chain" by T. K. Siu derives the integration-byparts formulas for functions of fundamental jump processes relating to a continuous-time, finite-state Markov chain using the Bismut measure change approach. The paper entitled "Generalized mutual synchronization between two controlled interdependent networks" by Q. Xu et al. focuses on the generalized mutual synchronization between two controlled interdependent networks. The paper entitled "Cascading dynamics of heterogenous scale-free networks with recovery mechanism" by $\mathrm{S}$. $\mathrm{Li}$ et al. defines five kinds of weighting strategies to assign the external resources for recovering the edges from cascading failures in heterogeneous scale-free networks. The paper entitled "Portfolio strategy of financial market with regime switching driven by geometric Lévy process" by L. Zhou and Z. Wang studies the problem of a portfolio strategy for financial market with regime switching driven by the geometric Lévy process. The paper entitled "Gsanular space reduction to a $\beta$ multigranulation fuzzy rough set" by J. Zhou et al. further generalizes $\beta$ multigranulation rough set to fuzzy environment. The paper entitled "Boundary recognition by simulating a diffusion process in wireless sensor networks" by D. Gu et al. proposes a distributed algorithm for boundary recognition in wireless sensor networks.

Although the selected topics and published papers are not a comprehensive representation of stochastic Markovian jumping systems, the authors represent the rich and manyfaceted knowledge and we still hope the reader will find our special issue very useful.

\section{Acknowledgments}

We first express our great appreciation to all the authors of this special issue for their high quality contributions. All the reviewers' efforts in reviewing the papers are also very greatly acknowledged.

Shuping He Zhengguang $W u$ Hao Shen Yanyan Yin Quanxin Zhu 


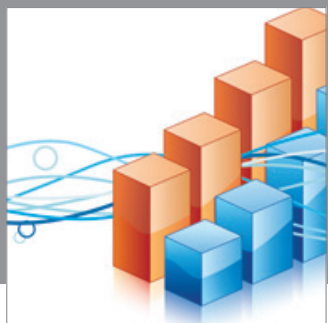

Advances in

Operations Research

mansans

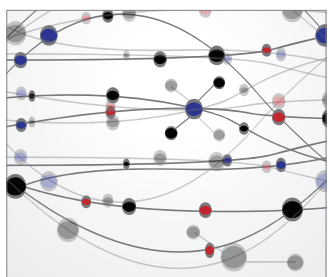

The Scientific World Journal
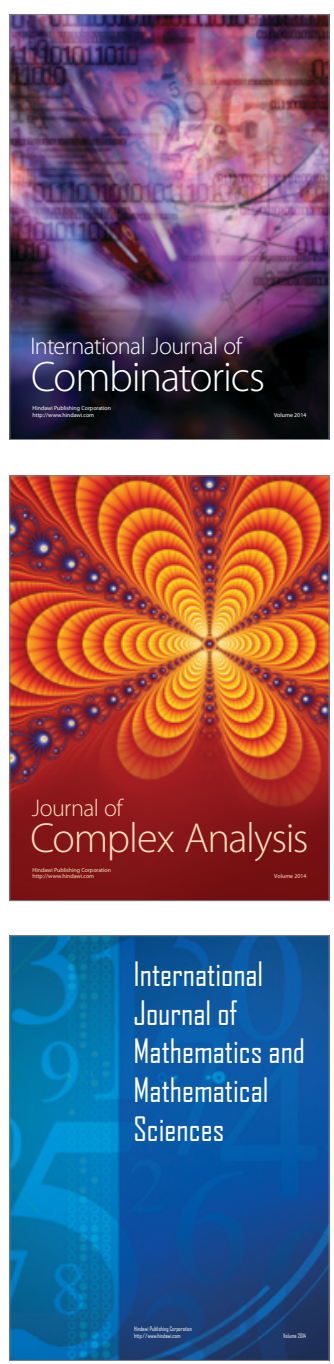
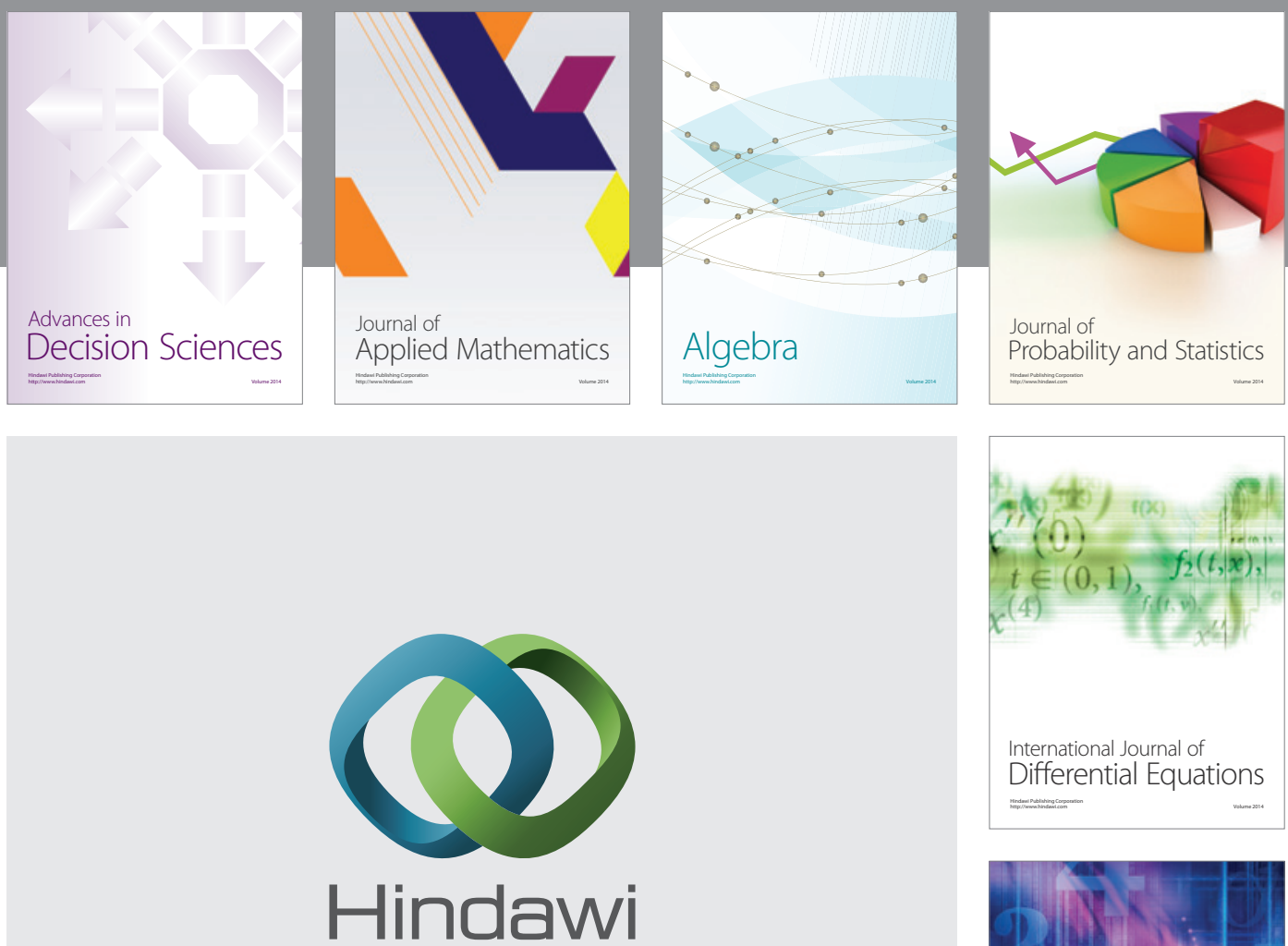

Submit your manuscripts at http://www.hindawi.com
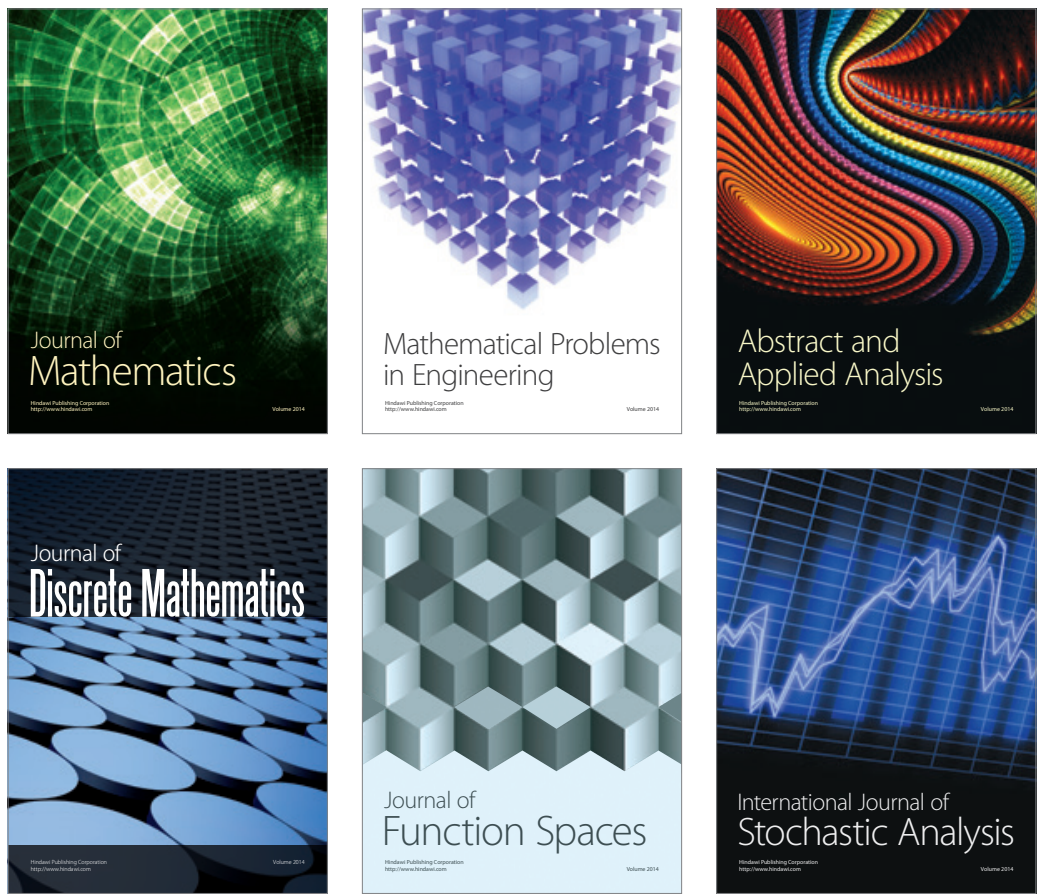

Journal of

Function Spaces

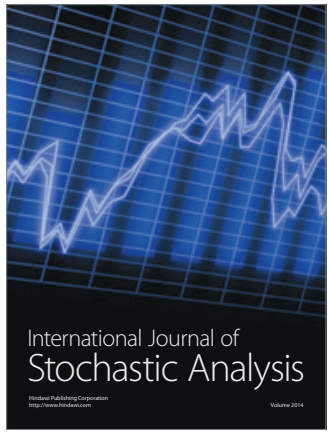

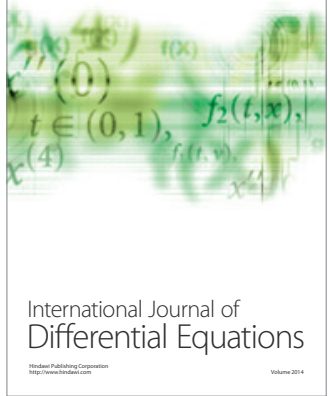
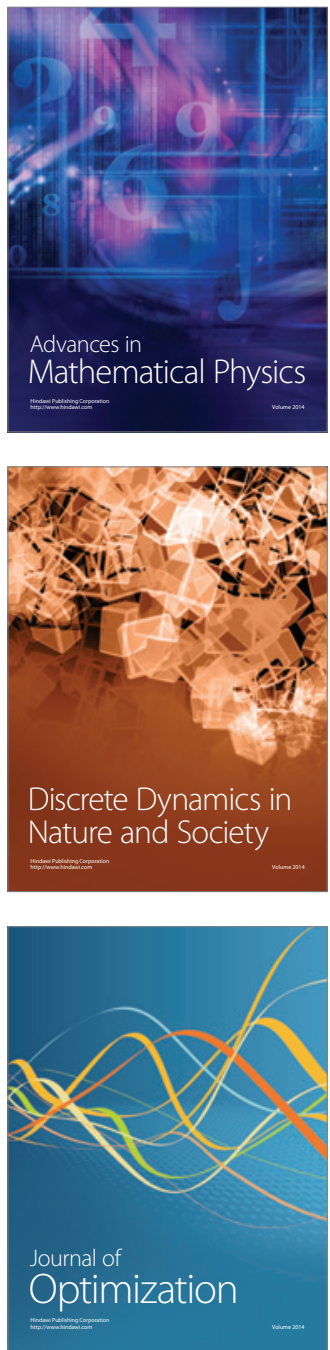